\title{
Separable degree of the Gauss map and strict dual curves over finite fields
}

\author{
Nazar Arakelian \\ Centro de Matemática, Computação e Cognição - Universidade Federal do ABC \\ Av. dos Estados 5001, CEP 09210-580, Santo André-SP, Brazil \\ n.arakelian@ufabc.edu.br
}

May 27, 2019

\begin{abstract}
Let $\mathcal{X}$ be a projective algebraic curve and denote by $\mathcal{X}^{\prime}$ its strict dual curve. The map $\gamma: \mathcal{X} \longrightarrow \mathcal{X}^{\prime}$ is called (strict) Gauss map of $\mathcal{X}$. In this manuscript, we study the separable degree of the Gauss map of curves defined over finite fields. In particular, we give a generalization of a known result on the separable degree of the Gauss map of plane Frobenius nonclassical curves. We also obtain a characterization of certain plane strange curves.
\end{abstract}

\section{Introduction}

For an algebraically closed field $\mathbb{K}$, let $\mathcal{X} \subset \mathbb{P}^{n}(\mathbb{K})$ be a (geometrically irreducible algebraic) curve defined over $\mathbb{K}$, where $\mathbb{P}^{n}(\mathbb{K})$ denotes the $n$-dimensional projective space defined over $\mathbb{K}$. If $\left(\mathbb{P}^{n}(\mathbb{K})\right)^{\prime}$ denotes the dual projective space, then the (strict) dual of $\mathcal{X}$, denoted by $\mathcal{X}^{\prime}$, is defined as the closure in $\left(\mathbb{P}^{n}(\mathbb{K})\right)^{\prime}$ of the set of osculating hyperplanes at the nonsingular locus of $\mathcal{X}$. The dual curve $\mathcal{X}^{\prime}$ is a subcover of $\mathcal{X}$, and the cover $\gamma: \mathcal{X} \longrightarrow \mathcal{X}^{\prime}$ is called the (strict) Gauss map of $\mathcal{X}$.

If the characteristic of $\mathbb{K}$ is 0 , then it is well known that $\gamma$ is birational. Moreover, if $\gamma^{\prime}: \mathcal{X}^{\prime} \longrightarrow \mathcal{X}^{\prime \prime}$ is the Gauss map of the dual curve $\mathcal{X}^{\prime}$, then $\gamma^{\prime} \circ \gamma$ is the identity map, i.e., $\mathcal{X}=\mathcal{X}^{\prime \prime}$, see 1 or 6 , Remark 2]. When $\mathbb{K}$ has characteristic $p>0$, the situation is the same as long as $p>n$ and $\mathcal{X}$ is a classical curve, that is, the intersection multiplicity of $\mathcal{X}$ and the osculating hyperplane at a generic point is $n$. In contrast, when $\mathcal{X}$ is nonclassical, then $\gamma$ is not necessarily separable and its separable degree can be positive. Indeed, in [6] several examples endorsing this facts are given. Furthermore, it follows as a particular case of a result of Kaji in [13] that, given a plane curve $\mathcal{C}$ and an arbitrary inseparable finite field extension $\mathbb{L} / \mathbb{K}(\mathcal{C})$, there exists a plane curve $\mathcal{X}$ such that $\gamma(\mathcal{X})=\mathcal{C}, \mathbb{K}(\mathcal{X}) \cong \mathbb{L}$ and the extension $\mathbb{K}(\mathcal{X}) / \mathbb{K}(\mathcal{C})$ coincides with $\mathbb{L} / \mathbb{K}(\mathcal{C})$, where $\mathbb{K}(\mathcal{C})$ (resp. $\mathbb{K}(\mathcal{X})$ ) denotes the function field of $\mathcal{C}$ (resp. $\mathcal{X}$ ) (see also $[5$ for a more general result). 
The properties of the Gauss map $\gamma$ and the dual $\mathcal{X}^{\prime}$ of a curve $\mathcal{X}$ over a field $\mathbb{K}$ of characteristic $p>0$ have been investigated by many authors in the last decades, sometimes either in a more general or a parallel context, see [5, 8, 9, 13. For instance, given a curve $\mathcal{X} \subset \mathbb{P}^{n}(\mathbb{K})$ the Gauss map $\iota^{(m)}$ : $\mathcal{X} \longrightarrow \mathbb{G}\left(\mathbb{P}^{n}(\mathbb{K}), m\right)$ of order $m(1 \leq m \leq n-1)$, which associates nonsingular points of $\mathcal{X}$ with the respective $m$-osculating spaces, where $\mathbb{G}\left(\mathbb{P}^{n}(\mathbb{K}), m\right)$ is the Grassmannian variety of $m$ spaces in $\mathbb{P}^{n}(\mathbb{K})$, is investigated by Homma and Kaji in [12] (note that $\iota^{(n-1)}=\gamma$ ). More precisely, if $\left(\varepsilon_{0}, \ldots, \varepsilon_{n}\right)$ denotes the order-sequence of $\mathcal{X}$, i.e., the sequence of all the possible intersection multiplicities of $\mathcal{X}$ with a hyperplane in $\mathbb{P}^{n}(\mathbb{K})$ at a generic point, [12, Theorem 1] states that the inseparable degree of $\iota^{(m)}$ is the highest power of $p$ dividing $\varepsilon_{m+1}$. In particular, if $\varepsilon_{n}=k p^{r}$, where $k$ is prime to $p$, then the inseparable degree of the Gauss map $\gamma$ is $p^{r}$.

In several situations, the computation of the separable degree of the Gauss map is desirable, specially when $\mathbb{K}$ is the algebraic closure of a finite field. For example, in the proof of the Natural Embedding Theorem for maximal curves, in [14, Section 3]. Also in [10, Section 3.3] to obtain bounds on the size of complete arcs, among others. In this direction, the following Theorem is a consequence of a result by Hefez and Voloch on Frobenius nonclassical curves [9, Proposition 3]. They actually were investigating Gaussian duals of such curves, to say, the closure of the image of the nonsingular locus of $\mathcal{X}$ by the Gauss map in the Grassmannian of lines in $\mathbb{P}^{n}(\mathbb{K})$. Hence, for $n=2$, the strict dual and the Gaussian dual are the same.

Theorem 1.1 (Hefez-Voloch). Let $\mathcal{X}$ be a plane curve defined over a finite field $\mathbb{F}_{q}$. If the image of infinitely many points of $P \in \mathcal{X}$ by the $\mathbb{F}_{q}$-Frobenius map belongs to the tangent line to $\mathcal{X}$ at $P$ (that is, $\mathcal{X}$ is $\mathbb{F}_{q}$-Frobenius nonclassical), then the Gauss map of $\mathcal{X}$ is purely inseparable.

In [6], Garcia and Voloch presented several relations between the osculating spaces of a curve $\mathcal{X}$ and the ones of its dual $\mathcal{X}^{\prime}$. As a consequence, they establish some criteria for when the equality $\mathcal{X}=\mathcal{X}^{\prime \prime}$ holds, and so when $\gamma$ has separable degree equals to 1 , provided that $\mathcal{X}^{\prime}$ is non-degenerate. Furthermore, they give a different proof of Theorem 1.1, and show that the converse holds for some extension of $\mathbb{F}_{q}$, with the assumption that $\mathcal{X}$ is smooth and nonclassical [6, Theorem 4].

Related to this subject, we have the so called strange curves. A curve $\mathcal{X}$ is called strange if all tangent lines to nonsingular points of $\mathcal{X}$ are concurrent. In this case, the point $P$ in which all generic tangent to $\mathcal{X}$ meet is called nucleus of $\mathcal{X}$. Note that if $\mathcal{X}$ is a plane curve, this is equivalent to the dual curve $\mathcal{X}^{\prime}$ be a line. The properties of strange curves have been broadly investigated over the last years. For instance, in [2] many properties of such curves are established, such as general shape of their equation and their genera.

In this paper, we basically study some maps on $\mathcal{X}$ that induce maps on $\mathcal{X}^{\prime}$, namely the Frobenius map and linear automorphisms. As a byproduct, we will provide a generalization of Theorem 1.1 for certain Frobenius nonclassical curves defined over $\mathbb{F}_{q}$. It also will be shown that for a curve defined over $\overline{\mathbb{F}}_{q}$, if $\overline{\mathbb{F}}_{q}\left(\mathcal{X}^{\prime}\right)$ is a subfield of a Galois subcover of $\overline{\mathbb{F}}_{q}(\mathcal{X})$ by a linear automorphism group, then $\mathcal{X}^{\prime}$ is degenerate, 
and some consequences of this fact will be exploited. This results will be obtained in Sections 3 and 4, after we establish some preliminary results and notation in Section 2.

\section{Background and notation}

For a prime number $p$, let $\mathbb{F}_{q}$ be the finite field of order $q$, where $q=p^{h}$ with $h>0$. Let $\mathcal{X} \subset \mathbb{P}^{n}\left(\overline{\mathbb{F}}_{q}\right)$ be a (geometrically irreducible algebraic) curve defined over $\mathbb{F}_{q}$, where $\overline{\mathbb{F}}_{q}$ denotes the algebraic closure of $\mathbb{F}_{q}$. Given an algebraic extension $\mathbb{H}$ of $\mathbb{F}_{q}$, the function field of $\mathcal{X}$ over $\mathbb{H}$ will be denoted by $\mathbb{H}(\mathcal{X})$. For a nonsingular point $P \in \mathcal{X}$, there exists a sequence $\left(j_{0}(P), \ldots, j_{n}(P)\right)$ of integers with $0 \leq j_{0}(P)<\cdots<$ $j_{n}(P)$, called order sequence of $P$, which is defined by all the possible intersection multiplicities of $\mathcal{X}$ and some hyperplane of $\mathbb{P}^{n}\left(\overline{\mathbb{F}}_{q}\right)$ at $P$. When there is no risk of confusion, $j_{i}$ denotes $j_{i}(P)$, for $i=0, \ldots, n$. It turns out that, except for finitely many points on $\mathcal{X}$, this sequence is the same, [16, Section 1]. It is called order sequence of $\mathcal{X}$, and denoted by $\left(\varepsilon_{0}, \ldots, \varepsilon_{n}\right)$. If $\left(\varepsilon_{0}, \ldots, \varepsilon_{n}\right)=(0, \ldots, n)$, the curve $\mathcal{X}$ is said to be a classical curve. Otherwise, $\mathcal{X}$ is nonclassical. Given a nonsingular point $P \in \mathcal{X}$, there exists an unique hyperplane $H_{P}(\mathcal{X}) \subset \mathbb{P}^{n}\left(\overline{\mathbb{F}}_{q}\right)$ such that the intersection multiplicity of $H_{P}(\mathcal{X})$ and $\mathcal{X}$ at $P$ is $j_{n}$. Such hyperplane is denoted by osculating hyperplane to $\mathcal{X}$ at $P$.

Let $t \in \overline{\mathbb{F}}_{q}(\mathcal{X})$ be a separating element and $k$ a non-negative integer. Given $f \in \overline{\mathbb{F}}_{q}(\mathcal{X})$, the $k$-th Hasse derivative of $f$ with respect to $t$ is denoted by $D_{t}^{(k)} f$. The following result is a consequence of [16, Theorem 1.1].

Proposition 2.1. Suppose that $\mathcal{X}$ is defined by the coordinate functions $x_{0}, \ldots, x_{n} \in \mathbb{F}_{q}(\mathcal{X})$. Let $P \in \mathcal{X}$ be a nonsingular point and let $t$ be a local parameter at $P$. The osculating hyperplane at $P$ is spanned by the points $\left(\left(D_{t}^{\left(j_{i}\right)} x_{0}\right)(P): \cdots:\left(D_{t}^{\left(j_{i}\right)} x_{n}\right)(P)\right)$, for $i=0, \ldots, n-1$.

Since $\mathcal{X}$ is defined over $\mathbb{F}_{q}$, i.e., $\mathcal{X}$ is defined by coordinate functions $x_{0}, \ldots, x_{n} \in \mathbb{F}_{q}(\mathcal{X})$, by [16, Proposition 2.1], there exist a sequence of non-negative integers $\left(\nu_{0}, \ldots, \nu_{n-1}\right)$, chosen minimally in the lexicographic order, such that

$$
\left|\begin{array}{ccc}
x_{0}^{q} & \cdots & x_{n}^{q} \\
D_{t}^{\left(\nu_{0}\right)} x_{0} & \cdots & D_{t}^{\left(\nu_{0}\right)} x_{n} \\
\vdots & \cdots & \vdots \\
D_{t}^{\left(\nu_{n-1}\right)} x_{0} & \cdots & D_{t}^{\left(\nu_{n-1}\right)} x_{n}
\end{array}\right| \neq 0 .
$$

This sequence is called $\mathbb{F}_{q}$-Frobenius order sequence of $\mathcal{X}$. It does not depend on $t$ and is invariant under change of projective coordinates of $\mathcal{X}$ (see [16, Proposition 1.4]). The curve $\mathcal{X}$ is called $\mathbb{F}_{q}$-Frobenius classical if $\left(\nu_{0}, \ldots, \nu_{n-1}\right)=(0, \ldots, n-1)$, and $\mathbb{F}_{q}$-Frobenius nonclassical otherwise. From [16, Proposition 2.1], there exists $I \in\{1, \ldots, n\}$ for which $\left\{\nu_{0}, \ldots, \nu_{n-1}\right\}=\left\{\varepsilon_{0}, \ldots, \varepsilon_{n}\right\} \backslash\left\{\varepsilon_{I}\right\}$. 
Let $\left(\mathbb{P}^{n}\left(\overline{\mathbb{F}}_{q}\right)\right)^{\prime}$ be the dual projective space and consider the open set

$$
\mathcal{X}^{\circ}=\left\{P \in \mathcal{X} \mid P \text { is nonsingular and } j_{i}(P)=\varepsilon_{i}, i=0, \ldots, n\right\} .
$$

In our context, by a generic point of $\mathcal{X}$ we mean a point $P \in \mathcal{X}^{\circ}$. The strict Gauss map $\gamma: \mathcal{X}^{\circ} \longrightarrow$ $\left(\mathbb{P}^{n}\left(\overline{\mathbb{F}}_{q}\right)\right)^{\prime}$ is the map defined at a generic point $P \in \mathcal{X}$ by $\gamma(P)=\left(H_{P}(\mathcal{X})\right)^{\prime}$, where $L^{\prime}$ denotes the dual of a hyperplane $L$. The closure of the image of $\gamma$ is the strict dual of $\mathcal{X}$, denoted by $\mathcal{X}^{\prime}$. For simplicity, we will denote the map $\mathcal{X} \longrightarrow \mathcal{X}^{\prime}$ also by $\gamma$. Note that $\gamma$ is a morphism defined in the nonsingular locus of $\mathcal{X}$. Here, we will abuse and call $\gamma$ a morphism of $\mathcal{X}$. The separable (respectively inseparable) degree of $\gamma$ will be denoted by $\operatorname{deg}_{s} \gamma$ (respectively $\operatorname{deg}_{i} \gamma$ ). The following facts concerning the map $\gamma$ (some of them pointed out in the introduction) hold:

- $\operatorname{deg}_{i} \gamma$ is the highest power of $p$ dividing $\varepsilon_{n}$ ([12, Theorem 1]).

- If $\mathcal{X}$ is classical and $p>n$, then $\operatorname{deg}_{i} \gamma=\operatorname{deg}_{s} \gamma=1$, and $\mathcal{X}=\mathcal{X}^{\prime \prime}(\underline{6})$.

- If $n=2$ and $\mathcal{X}$ is $\mathbb{F}_{q^{r}}$-Frobenius nonclassical for some $r>0$, then $\varepsilon_{2}$ is a power of $p, \operatorname{deg}_{s} \gamma=1$ and $\operatorname{deg}_{i} \gamma=\varepsilon_{2}$ ([9, Propositions 1, 3 and 4]).

- If $d=\operatorname{deg} \mathcal{X}$, then

$$
\operatorname{deg}_{s} \gamma \leq \frac{d}{\varepsilon_{n}}
$$

Indeed, by definition, $\operatorname{deg}_{s} \gamma$ is the number of genereic points of $\mathcal{X}$ sharing the same osculating hyperplane. Thus, (2.2) follows from Bézout's Theorem.

For a given $r>0$, the $\mathbb{F}_{p^{r}}$-Frobenius map $\Phi_{p^{r}}: \mathcal{X} \longrightarrow \mathbb{P}^{n}\left(\overline{\mathbb{F}}_{q}\right)$ is defined by $\Phi_{p^{r}}: P \mapsto P^{p^{r}}$. It induces a map from the function field $\overline{\mathbb{F}}_{q}(\mathcal{X})$ onto $\overline{\mathbb{F}}_{q}(\mathcal{X})^{p^{r}}=\left\{f^{p^{r}} \mid f \in \overline{\mathbb{F}}_{q}(\mathcal{X})\right\}$. Therefore, $\Phi_{p^{r}}$ is purely inseparable of degree $p^{r}$. We point out that although $\Phi_{q}$ is purely inseparable, we have $\Phi_{q}: \mathcal{X} \longrightarrow \mathcal{X}$, as $\mathcal{X}$ is defined over $\mathbb{F}_{q}$.

Suppose that $p>n$ and $\varepsilon_{n}=p^{r}$ for some $r>0$. If $\mathcal{X}$ is defined by the coordinate functions $x_{0}, \ldots, x_{n}$, then [11, Theorem 7.65] ensures that there exist $z_{0}, \ldots, z_{n} \in \mathbb{F}_{q}(\mathcal{X})$ such that $z_{i}$ is a separating element for at least one $i$ and

$$
z_{0}^{p^{r}} x_{0}+\cdots+z_{n}^{p^{r}} x_{n}=0
$$

Furthermore, for a generic point $P \in \mathcal{X}$, the osculating hyperplane to $\mathcal{X}$ at $P$ is defined by

$$
H_{P}(\mathcal{X}):\left(z_{0}^{p^{r}}(P)\right) X_{0}+\cdots+\left(z_{n}^{p^{r}}(P)\right) X_{n}=0
$$

Therefore, in such case we conclude that $\gamma=\Phi_{p^{r}} \circ \gamma_{s}=\left(z_{0}^{p^{r}}: \cdots: z_{n}^{p^{r}}\right)$, where $\gamma_{s}=\left(z_{0}: \cdots: z_{n}\right)$ is a separable morphism. In particular, $\operatorname{deg}_{s} \gamma=\operatorname{deg} \gamma_{s}$ and $\operatorname{deg}_{i} \gamma=\operatorname{deg} \Phi_{p^{r}}=p^{r}$. 


\section{Generalization of Theorem 1.1}

Let $\mathcal{X} \subset \mathbb{P}^{n}\left(\overline{\mathbb{F}}_{q}\right)$ a curve defined over $\mathbb{F}_{q}$. Let $\left(\varepsilon_{1}, \ldots, \varepsilon_{n}\right)$ and $\left(\nu_{0}, \ldots, \nu_{n-1}\right)$ denote the order sequence and $\mathbb{F}_{q}$-Frobenius order sequence of $\mathcal{X}$, respectively. Assume that $\nu_{n-1}=\varepsilon_{n}$. Then $\left(\nu_{0}, \ldots, \nu_{n-1}\right) \neq$ $\left(\varepsilon_{0}, \ldots, \varepsilon_{n-1}\right)$, and so $\mathcal{X}$ is $\mathbb{F}_{q}$-Frobenius nonclassical. Moreover, from Proposition 2.1 and (2.1), the hypothesis $\nu_{n-1}=\varepsilon_{n}$ is equivalent to the following: $\Phi_{q}(P) \in H_{P}(\mathcal{X})$ for a generic point $P \in \mathcal{X}$. Under such condition, we obtain a slight improvement of bound (2.2).

Proposition 3.1. Let $\mathcal{X} \subset \mathbb{P}^{n}\left(\overline{\mathbb{F}}_{q}\right)$ be a curve defined over $\mathbb{F}_{q}$ with order sequence $\left(\varepsilon_{0}, \ldots, \varepsilon_{n}\right)$. Let $1 \leq \ell<n$ and suppose that $\mathcal{X}$ is $\mathbb{F}_{q^{i}}$-Frobenius nonclassical for $i=1, \ldots, \ell$ such that the last term of the $\mathbb{F}_{q^{i}}$-Forbenius order sequence equals to $\varepsilon_{n}$. Then

$$
\operatorname{deg}_{s} \gamma \leq \frac{d}{\varepsilon_{n}+\ell}
$$

Proof. Given a generic point $P \in \mathcal{X}$ with osculating hyperplane $H_{P}(\mathcal{X})$, we have that for a fixed $i$, the osculating hyperplane at $\Phi_{q^{i}}(P)$ is $\Phi_{q^{i}}\left(H_{P}(\mathcal{X})\right)$. Note that, since $P$ is generic, its osculating hyperplane is not $\mathbb{F}_{q^{i}}$-rational, and then $\Phi_{q^{i}}\left(H_{P}(\mathcal{X})\right) \neq H_{P}(\mathcal{X})$. Hence, to each generic point sharing the same osculating hyperplane $H_{P}(\mathcal{X})$, we have an extra point in $H_{P}(\mathcal{X}) \cap \mathcal{X}$ for which $H_{P}(\mathcal{X})$ is not the osculating hyperplane, namely $\Phi_{q^{i}}(P)$. Since $\Phi_{q^{i}}$ is injective, all these points are distinct. Finally, if $Q \in \mathcal{X}$ is such that $H_{P}(\mathcal{X})$ is osculating to $\mathcal{X}$ at $Q$, then $\Phi_{q^{i}}(P) \neq \Phi_{q^{j}}(Q)$ if $i \neq j$, with $1 \leq i<j \leq \ell$. Indeed, if $\Phi_{q^{i}}(P)=\Phi_{q^{j}}(Q)$, then $\Phi_{q^{j-i}}(Q)=P$, and so $H_{P}(\mathcal{X})$ is fixed by $\Phi_{q^{j-i}}$, a contradiction as $P \in \mathcal{X}$ is generic. Therefore (3.1) follows from the Bézout's Theorem.

However, note that the result in Theorem 1.1] does not depend on the degree of the curve. In fact, it only depends on the dimension of the ambient projective space. In what follows, $\lceil m\rceil$ denotes the smallest integer bigger or equal to the rational number $m$.

Theorem 3.2. Let $\mathcal{X} \subset \mathbb{P}^{n}\left(\overline{\mathbb{F}}_{q}\right)$ be a curve defined over $\mathbb{F}_{q}$ with order sequence $\left(\varepsilon_{0}, \ldots, \varepsilon_{n}\right)$. Let $1 \leq \ell<n$ and suppose that $\mathcal{X}$ is $\mathbb{F}_{q^{i}}$-Frobenius nonclassical for $i=1, \ldots, \ell$ such that the last term of the $\mathbb{F}_{q^{i}}$ Forbenius order sequence equals to $\varepsilon_{n}$. If

$$
\operatorname{deg}_{s} \gamma>\left\lceil\frac{n}{\ell}-1\right\rceil
$$

then for a generic $P \in \mathcal{X}$, the set $S(P)=\left\{\Phi_{q^{k}}(Q) \mid Q \in \gamma^{-1}(\gamma(P)), k=0, \ldots, \ell-1\right\}$ lies in a linear subspace of $\mathbb{P}^{n}\left(\overline{\mathbb{F}}_{q}\right)$ of dimension $n-2$.

Proof. Suppose that $\operatorname{deg}_{s} \gamma>\left\lceil\frac{n}{\ell}-1\right\rceil$. Let $P \in \mathcal{X}$ be a generic point and assume that $S(P)$ is not in a linear subspace of $\mathbb{P}^{n}\left(\overline{\mathbb{F}}_{q}\right)$ of dimension $n-2$. The hypothesis on the $\mathbb{F}_{q^{i}}$-Frobenius order sequence gives that $\Phi_{q^{i}}(P) \in H_{P}(\mathcal{X})$ for all $i=1, \ldots, \ell$. Since $\operatorname{deg}_{s} \gamma>\left\lceil\frac{n}{\ell}-1\right\rceil$, there are at least $\lceil n / \ell\rceil$ points sharing the same osculating hyperplane $H_{P}(\mathcal{X})$. Then, as $S(P) \subset H_{P}(\mathcal{X})$ and it is not in a linear 
subspace of dimension $n-2$, we have for sure $n$ points of $S(P)$ in general position in $H_{P}(\mathcal{X})$, and $H_{P}(\mathcal{X})$ is completely determined by these points. Hence $\Phi_{q}\left(H_{P}(\mathcal{X})\right)$ is completely determined by the set $\left\{\Phi_{q}(R) \mid R \in S(P)\right\}$. But if $R \in S(P)$, we have $R=\Phi_{q^{k}}(Q)$ for some $Q \in \gamma^{-1}(\gamma(P))$ and $k=0, \ldots, \ell-1$, thus $\Phi_{q}(R)=\Phi_{q^{k+1}}(Q) \in H_{P}(\mathcal{X})$. Therefore, $\Phi_{q}\left(H_{P}(\mathcal{X})\right)=H_{P}(\mathcal{X})$. Since $P$ is generic, this implies that $\Phi_{q}$ fixes infinitely many points in $\mathcal{X}^{\prime}$, that is, $\mathcal{X}^{\prime}$ has infinitely many $\mathbb{F}_{q^{-}}$-rational points, which is a contradiction.

In particular, for $\ell=1$ we have the following.

Theorem 3.3. Let $\mathcal{X} \subset \mathbb{P}^{n}\left(\overline{\mathbb{F}}_{q}\right)$ be an $\mathbb{F}_{q}$-Frobenius nonclassical curve defined over $\mathbb{F}_{q}$ such that $\nu_{n-1}=$ $\varepsilon_{n}$. If $\operatorname{deg}_{s} \gamma \geq n$ then the generic pre-image of $\gamma$ lies in a linear subspace of $\mathbb{P}^{n}\left(\overline{\mathbb{F}}_{q}\right)$ of dimension $n-2$.

Remark 3.4. If $n=2$, then the hypothesis $\nu_{n-1}=\varepsilon_{n}$ is equivalent to $\mathcal{X}$ being $\mathbb{F}_{q}$-Frobenius nonclassical. Moreover, a linear subspace in $\mathbb{P}^{2}\left(\overline{\mathbb{F}}_{q}\right)$ of dimension $n-2$ is just a point. Hence, we conclude from Theorem 3.3 that $\operatorname{deg}_{s} \gamma=1$. In other words, we obtain the result stated in Theorem 1.1 .

To illustrate Theorem 3.3. we now show how to construct examples of curves in $\mathbb{P}^{3}\left(\overline{\mathbb{F}}_{q}\right)$ with $\operatorname{deg}_{s} \gamma=k$, with $k$ prime to $p$, such that the points in a generic pre-image of $\gamma$ are collinear. Let $\mathcal{F}: z_{0}^{p^{r}} x+$

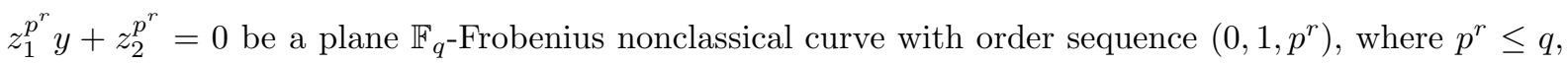
$z_{0}, z_{1}, z_{2} \in \mathbb{F}_{q}(\mathcal{F})=\mathbb{F}_{q}(x, y)$ and $x$ is a separating variable. Then

$$
D_{x}^{(1)}(y)=\frac{y-y^{q}}{x-x^{q}}=-\frac{z_{0}^{p^{r}}}{z_{1}^{p^{r}}}
$$

and $D_{x}^{(l)}(y)=0$ for $1<l<p^{r}$. For $k>1$ prime to $p$, let $f \in \overline{\mathbb{F}}_{q}(x, y)$ such that $f \neq w^{d}$ for all $w \in \mathbb{F}_{q}(x, y)$ and $d>1$ divisor of $k$ (for instance, we may chose $f=1 / t$, where $t$ is a local parameter at some nonsingular point $P \in \mathcal{F})$. Suppose that $z$ is a root of $T^{k}-f \in \overline{\mathbb{F}}_{q}(x, y)[T]$ such that $D_{x}^{(2)}(z) \neq 0$ (note that $x$ is also separating in $\overline{\mathbb{F}}_{q}(x, y, z)$ ). In particular,

$$
D_{x}^{(1)}(z) \neq \frac{z-z^{q}}{x-x^{q}}
$$

Consider $\mathcal{X}=\phi(\mathcal{F}) \subset \mathbb{P}^{3}\left(\overline{\mathbb{F}}_{q}\right)$, where $\phi=(1: x: y: z)$. Since

$$
\left|\begin{array}{cccc}
1 & x & y & z \\
0 & 1 & D_{x}^{(1)}(y) & D_{x}^{(1)}(z) \\
0 & 0 & 0 & D_{x}^{(2)}(z) \\
0 & 0 & D_{x}^{(i)}(y) & D_{x}^{(i)}(z)
\end{array}\right|=-D_{x}^{(2)}(z) \cdot D_{x}^{(i)}(y)
$$

for $i>1$, we conclude that the order sequence of $\mathcal{X}$ is $\left(0,1,2, p^{r}\right)$. Futhermore, we have $\nu_{2}=\varepsilon_{3}$, since for $j>1$ the equality 


$$
\left|\begin{array}{cccc}
1 & x^{q} & y^{q} & z^{q} \\
1 & x & y & z \\
0 & 1 & D_{x}^{(1)}(y) & D_{x}^{(1)}(z) \\
0 & 0 & D_{x}^{(j)}(y) & D_{x}^{(j)}(z)
\end{array}\right|=D_{x}^{(j)}(y) \cdot\left(D_{x}^{(j)}(z)\left(x-x^{q}\right)-\left(z-z^{q}\right)\right)
$$

gives that the $\mathbb{F}_{q}$-Frobenius order sequence of $\mathcal{X}$ is $\left(0,1, p^{r}\right)$. Therefore, we conclude from Proposition 2.1 that the Gauss map of $\mathcal{X}$ is given by $\gamma=\left(z_{2}^{p^{r}}: z_{0}^{p^{r}}: z_{1}^{p^{r}}: 0\right)$. Since $\mathcal{F}$ is $\mathbb{F}_{q}$-Frobenius nonclassical, we have that the morphism $\left(z_{0}: z_{1}: z_{2}\right)$ is birational, and so $\gamma(\mathcal{X})=\mathcal{X}^{\prime}$ is birationally equivalent to $\mathcal{F}$ up to the $\Phi_{p^{r}}$-Frobenius map. In particular, $\operatorname{deg}_{s} \gamma=k$. Finally, if $\lambda \in \overline{\mathbb{F}}_{q}$ denotes a $k$-primitive root of unity, we clearly have that the points $\left(1: a: b: \lambda^{j} c\right) \in \mathcal{X}$ with $j=0, \ldots, k-1$ have the same osculating hyperplane and are collinear.

Example 3.5. Let $q$ be an even power of $p>2$ and set $\mathcal{F}: x^{\sqrt{q}+1}+y^{\sqrt{q}+1}+1=0$ defined over $\mathbb{F}_{q}$ $\left(\right.$ Hermitian curve over $\mathbb{F}_{q}$ ). It is well known that $\mathcal{F}$ is $\mathbb{F}_{q}$-Frobenius nonclassical, see [7, Theorem 2] for instance. For $k>1$ prime to $p$, the function $f=\frac{x^{2}}{y}$ is such that $f \neq w^{d}$ for all $w \in \overline{\mathbb{F}}_{q}(x, y)$ and $d$ divisor of $k$, as there exists a place $Q$ of $\overline{\mathbb{F}}_{q}(x, y)$ such that $v_{Q}(f)=-1$ ([15, Corollary 3.7.4]). Let $z$ be such that $z^{k}=\frac{x^{2}}{y}$. One can show that $D_{x}^{(2)}(z) \neq 0$. Hence, by the discussion above, $\phi(\mathcal{F}) \subset \mathbb{P}^{3}\left(\overline{\mathbb{F}}_{q}\right)$, where $\phi=(1: x: y: z)$ is such that $\varepsilon_{3}=\nu_{2}=\sqrt{q}, \operatorname{deg}_{s} \gamma=k$ and the generic points of $\mathcal{X}$ sharing the same osculating hyperplane are collinear.

One clearly has that the result of Theorem 3.3 does not hold without the assumption $\nu_{n-1}=\varepsilon_{n}$. For instance, as discussed previously in this paper, it is known that if a plane curve $\mathcal{X}$ is nonsingular and $\operatorname{deg}_{s} \gamma>1$, then $\mathcal{X}$ is Frobenius classical. For sake of completeness, we will present the following example for curves in higher dimensional spaces. It is based on [6, Example 5].

Example 3.6. Consider a curve $\mathcal{F}$ defined by $x^{6}=f(y)$ over $\mathbb{F}_{p}$, where $f(T) \in \mathbb{F}_{p}[T]$, with $p>3$ and set $\mathcal{X}=\psi(\mathcal{F}) \subset \mathbb{P}^{3}\left(\overline{\mathbb{F}}_{p}\right)$, where $\psi=\left(1: x: x^{2}: x^{3 p}+y^{p} x+x^{6 p+2}\right)$. Then $\gamma=\left(x^{3 p}: y^{p}: x^{6 p}:-1\right)$, and so $\operatorname{deg}_{s} \gamma=3$. Moreover, a generic pre-image of $\gamma$ is not collinear. Indeed, let $P_{1}=\left(1: a: a^{2}\right.$ : $\left.a^{3 p}+b^{p} a+a^{6 p+2}\right) \in \mathcal{X}$. Then $P_{2}=\left(1: \xi a:(\xi a)^{2}: a^{3 p}+b^{p}(\xi a)+(\xi a)^{6 p+2}\right)$ and $P_{3}=\left(1: \xi^{2} a:\left(\xi^{2} a\right)^{2}:\right.$ $\left.a^{3 p}+b^{p}\left(\xi^{2} a\right)+\left(\xi^{2} a\right)^{6 p+2}\right)$ have the same image $\left(a^{3 p}: b^{p}: a^{6 p}:-1\right)$ under $\gamma$, where $\xi$ is a primitive cubic root of 1 . However, one can easily check that such points are not collinear.

We can also specialize Theorem 3.2 in the case $\ell=n-1$.

Theorem 3.7. Let $\mathcal{X} \subset \mathbb{P}^{n}\left(\overline{\mathbb{F}}_{q}\right)$ be a curve defined over $\mathbb{F}_{q}$ with order sequence $\left(\varepsilon_{0}, \ldots, \varepsilon_{n}\right)$. Suppose that $\mathcal{X}$ is $\mathbb{F}_{q^{i}}$-Frobenius nonclassical for $i=1, \ldots, n-1$ such that the last term of the $\mathbb{F}_{q^{i}}$-Forbenius order sequence equals to $\varepsilon_{n}$. Then $\operatorname{deg}_{s} \gamma=1$, that is, the Gauss map $\gamma$ is purely inseparable.

Proof. The case $n=2$ is discussed in Remark 3.4. Assume that $n>2$ and $\operatorname{deg}_{s} \gamma>1$. Let $P \in \mathcal{X}$ be a generic point and let $Q \in \mathcal{X}$ such that $P \neq Q$ and $H_{P}(\mathcal{X})=H_{Q}(\mathcal{X})$. Denote by $d$ the dimension of 
the linear subspace $L \subset \mathbb{P}^{n}\left(\overline{\mathbb{F}}_{q}\right)$ defined by the set $\left\{P, \Phi_{q}(P), \ldots, \Phi_{q^{n-2}}(P), Q, \Phi_{q}(Q), \ldots, \Phi_{q^{n-2}}(Q)\right\}$. By Theorem 3.2, one has $d \leq n-2$. Suppose that $d<n-2$. Then the set $A=\left\{P, \Phi_{q}(P), \ldots, \Phi_{q^{n-2}}(P)\right\}$ determines a linear subspace $\tilde{L}$ of dimension $k \leq d<n-2$ and $\Phi_{q}(R) \in A \subset \tilde{L}$ for all $R=\phi_{q^{j}}(P)$, $j=0, \ldots, n-3$. Note that since $A$ has $n-1$ points, we have a redundancy of points to generate $\tilde{L}$. If $\tilde{L}$ is defined by the set $A \backslash\left\{\Phi_{q^{n-2}}(P)\right\}$, then $\tilde{L}$ is fixed by $\Phi_{q}$, which gives that there are infinitely many linear subspaces of degree $k$ defined over $\mathbb{F}_{q}$, a contradiction. If $\tilde{L}$ is not defined by $A \backslash\left\{\Phi_{q^{n-2}}(P)\right\}$, then the subspace determined by such set of points has dimension smaller than $k$, and by induction we arrive again at a contradiction

Suppose then $d=n-2$. In this case, we may assume that $L$ is defined by the set $A$, because otherwise $A$ would determine a linear subspace of dimension smaller then $n-2$ and we would obtain a contradiction proceeding as above. If $B=\left\{Q, \Phi_{q}(Q), \ldots, \Phi_{q^{n-3}}(Q)\right\}$ is not contained in the linear subspace determined by $C=\left\{P, \Phi_{q}(P), \ldots, \Phi_{q^{n-3}}(P)\right\}$, then there exists $j \in\{0, \ldots, n-3\}$ such that $L$ is defined by $C \cup\left\{\Phi_{q^{j}}(Q)\right\}$. Therefore, $L$ is fixed by $\Phi_{q}$, which is a contradiction. If $B$ is contained in the the linear subspace determined by $C$, an inductive argument as used previously also gives rise to a contradiction. Hence $\operatorname{deg}_{s} \gamma=1$, as claimed.

Example 3.8. Suppose $p>3$ and consider the irreducible plane curve $\mathcal{F}$ defined by the affine equation

$$
y^{q}-y=\frac{1-x^{q-1}}{1+x^{q-1}}
$$

over $\mathbb{F}_{q}$. Then $x$ is a separating element of $\mathbb{F}_{q}(\mathcal{F})=\mathbb{F}_{q}(x, y)$. Let $z$ be a root of the polynomial

$$
T-T^{q}+\left(x-x^{q}\right) x+\left(y-y^{q}\right) x^{2} \in \mathbb{F}_{q}(x, y)[T]
$$

and denote by $\mathcal{C}$ the curve defined over $\mathbb{F}_{q}$ such that $\mathbb{F}_{q}(\mathcal{C})=\mathbb{F}_{q}(x, y, z)$. Note that $x$ is separating in $\mathbb{F}_{q}(\mathcal{C})$. Thus the following equations are satisfied:

$$
\begin{cases}\left(z-z^{q}\right)+\left(x-x^{q}\right) x+\left(y-y^{q}\right) x^{2} & =0, \\ \left(z-z^{q^{2}}\right)+\left(x-x^{q^{2}}\right) x^{q}+\left(y-y^{q^{2}}\right) x^{2 q} & =0 .\end{cases}
$$

Finally, let $\psi=\left(1: x: x^{2}: z^{q}+x^{q+1}+y^{q} x^{2}\right): \mathcal{C} \longrightarrow \mathbb{P}^{3}\left(\overline{\mathbb{F}}_{q}\right)$ and define $\mathcal{X}=\psi(\mathcal{C})$. The order sequence of $\mathcal{X}$ is $(0,1,2, q)$. By the equations above we conclude that the $\mathbb{F}_{q}$ and $\mathbb{F}_{q^{2}}$-Frobenius order sequences are the same, namely $(0,1, q)$. Therefore $\mathcal{X}$ satisfies the hypotheses of Theorem 3.7 .

We end this section by pointing out that if we do not require that $i=1, \ldots, n-1$ in Theorem 3.7 then the result does not necessarily holds. To this end, we present two examples of curves in $\mathbb{P}^{3}\left(\overline{\mathbb{F}}_{q}\right)$ (based on the construction given in page 6) satisfying the hypothesis of Theorem 3.7 except that this time we do not have $i=1,2$, such that one has purely inseparable Gauss map and the other does not. 
Example 3.9. Suppose $p>3$. Let $u, m$ be co-prime positive integers with $m>2$ and $m>u$, and consider the Borges curve defined over $\mathbb{F}_{q}$ by $\mathcal{F}: f(x, y)=0$, where

$$
f(x, y)=\frac{\left(x^{q^{u}}-x\right)\left(y^{q^{m}}-y\right)-\left(x^{q^{m}}-x\right)\left(y^{q^{u}}-y\right)}{\left(x^{q^{2}}-x\right)\left(y^{q}-y\right)-\left(x^{q}-x\right)\left(y^{q^{2}}-y\right)}
$$

This curve was introduced in [3], and it was proved that it is the only simultaneously $\mathbb{F}_{q^{m}-\text { and }} \mathbb{F}_{q^{u-}}$ Frobenius nonclassical curve for the morphism of lines ([3, Theorem 1.1]), and its order sequence is $\left(0,1, q^{u}\right)$.

Let $\mathcal{X}=\psi(\mathcal{F})$, where $\psi=(1: x: y: x y)$. Since $D_{x}^{(2)}(x y)=y+x D_{x}^{(1)}(y) \neq 0$, we have that the order sequence of $\mathcal{X}$ is $\left(0,1,2, q^{u}\right)$ and the $\mathbb{F}_{q^{r}}$-Frobenius order sequence is $\left(0,1, q^{u}\right)$ for $r=u, m$. Thus $\gamma$ is purely inseparable, as $\overline{\mathbb{F}}_{q}(\mathcal{X})=\overline{\mathbb{F}}_{q}(\mathcal{F})$ (recall that in such situation we have $\overline{\mathbb{F}}_{q}\left(\gamma_{s}(\mathcal{X})\right)=\overline{\mathbb{F}}_{q}\left(\gamma_{s}(\mathcal{F})\right)=$ $\left.\overline{\mathbb{F}}_{q}(\mathcal{F})\right)$.

Now let $P=(a: b: 1) \in \mathcal{F}$ be a generic point such that $x-a$ is a local parameter at $P$ (is not so difficult to check that there exists such point). Let $k>1$ be an integer such that $p \nmid k(k+1)$. Set $w=\frac{1}{x-a}$, and let $z$ be a root of $T^{k}-w$. Define $\varphi=(1: x: y: z): \mathcal{F} \longrightarrow \mathbb{P}^{3}\left(\overline{\mathbb{F}}_{q}\right)$ and set $\mathcal{Y}=\varphi(\mathcal{F})$. Thus $\left[\overline{\mathbb{F}}_{q}(\mathcal{Y}): \overline{\mathbb{F}}_{q}(\mathcal{F})\right]=k$. The condition $p \nmid k+1$ ensures that $D_{x}^{(2)}(z) \neq 0$. Hence the order sequence of $\mathcal{Y}$ is $\left(0,1,2, q^{u}\right)$, and $\mathcal{Y}$ is $\mathbb{F}_{q^{r}}$-Frobenius nonclassical, for $r=u, m$. However, one has $\operatorname{deg}_{s} \gamma=k>1$.

\section{Strict dual curves covered by a quotient curve}

Let $\mathcal{X} \subset \mathbb{P}^{n}\left(\overline{\mathbb{F}}_{q}\right)$ be a curve defined over $\mathbb{F}_{q}$ equipped with the Frobenius map $\Phi_{q}$. In Section 3 we took advantage of the fact that $\Phi_{q}$ induces a Frobenius map on $\mathcal{X}^{\prime}$ such that $\gamma \circ \Phi_{q}=\Phi_{q} \circ \gamma$, to bound $\operatorname{deg}_{s} \gamma$. In this section, we use a similar approach for linear automorphisms.

Let $\left(\varepsilon_{0}, \ldots, \varepsilon_{n}\right)$ the order sequence of $\mathcal{X}$ and assume that $\varepsilon_{n}=p^{r}$, where $n>p$. Recall that in such case, $\gamma=\Phi_{p^{r}} \circ \gamma_{s}$, and the extension $\overline{\mathbb{F}}_{q}(\mathcal{X}) / \overline{\mathbb{F}}_{q}\left(\gamma_{s}(\mathcal{X})\right)$ is separable. In this section, we will investigate what happens if there exists an intermediate field $\overline{\mathbb{F}}_{q}\left(\gamma_{s}(\mathcal{X})\right) \subseteq \mathbb{F} \subsetneq \overline{\mathbb{F}}_{q}(\mathcal{X})$ such that the extension $\overline{\mathbb{F}}_{q}(\mathcal{X}) / \mathbb{F}$ is Galois defined by a linear automorphism group $G$, specially when $\mathcal{X}$ is a plane curve. In what follows, $\operatorname{Aut}(\mathcal{X})$ will denote the full automorphism group of $\mathcal{X}$.

Proposition 4.1. Let $\mathcal{X} \subset \mathbb{P}^{n}\left(\overline{\mathbb{F}}_{q}\right)$ defined over $\mathbb{F}_{q}$ such that $\varepsilon_{n}=p^{r}$, where $n>p$. If $\gamma_{s}(\mathcal{X})$ is a subcover of a quotient curve $\mathcal{X} / G$, for some linear nontrivial subgroup $G<\operatorname{Aut}(\mathcal{X})$, then $\mathcal{X}^{\prime}$ is degenerate. In particular, if $\mathcal{X}$ is a plane curve, then $\mathcal{X}$ is strange.

Proof. Since $G$ is linear, $G$ induces a linear automorphism group in $\mathcal{X}^{\prime}$, as for a nontrivial $\sigma \in G$ and a generic point $P \in \mathcal{X}$, one has $H_{\sigma(P)}(\mathcal{X})=\sigma\left(H_{P}(\mathcal{X})\right)$. Since $\mathcal{X}^{\prime}$ differs from $\gamma_{s}(\mathcal{X})$ by a Frobenius colineation, one has an induced linear action by $G$ in $\gamma_{s}(\mathcal{X})$. Let $P \in \mathcal{X}$ be a generic point. Then the orbit of $P$ by $G$, namely $G(P)$, lies over the same point $Q \in \mathcal{X} / G$. Note that since $G$ is nontrivial, we have $\# G(P)>1$. In its turn, $Q$ lies over a point $\tilde{P} \in \gamma_{s}(\mathcal{X})$. But $\tilde{P}$ corresponds to the osculating hyperplane of all point in $G(P)$, and then $Q$ is fixed by the action induced by $G$. This implies that the 
group $G$ acting on $\gamma_{s}(\mathcal{X})$ has infinite fixed points. Hence, $G$ is trivial in $\operatorname{Aut}\left(\gamma_{s}(\mathcal{X})\right)$. Therefore, $\gamma_{s}(\mathcal{X})$ is contained in the linear subvariety fixed by $G$ in the dual projective space.

Remark 4.2. Recall that every automorphism in a nonsingular curve is linear.

We now will exploit the above situation for plane curves. If $p>2$ and $\mathcal{X}$ is a plane strange nonrational curve, then $\operatorname{deg}_{s} \gamma>1$, and so $\mathcal{X}$ is nonclassical. Hence, there are $z_{0}, z_{1}, z_{2} \in \overline{\mathbb{F}}_{q}(\mathcal{X})$ not all zero and $r>0$ such that

$$
z_{0}^{p^{r}} x+z_{1}^{p^{r}} y+z_{2}^{p^{r}}=0 .
$$

Here, $\operatorname{deg}_{i} \gamma=p^{r}$ and $\gamma_{s}=\left(z_{0}: z_{1}: z_{2}\right)$. Up to a projective transformation, we may assume that a generic tangent to $\mathcal{X}$ passes trough $(1: 0: 0)$. In other words, we may assume $z_{0}=0$, and $\mathcal{X}$ is defined by a polynomial dividing

$$
\left(Z_{1}(X, Y, Z)\right)^{p^{r}} Y+\left(Z_{2}(X, Y, Z)\right)^{p^{r}} Z
$$

where $Z_{i}(X, Y, Z) \in \overline{\mathbb{F}}_{q}[x, y, z]$ are homogeneous, $i=1,2$, and $z_{1}, z_{2}$ and $y$ are the projections of $Z_{1}, Z_{2}$ and $Y$ in $\overline{\mathbb{F}}_{q}(\mathcal{X})$, respectively.

Theorem 4.3. Let $\mathcal{X}$ be plane curve defined over $\mathbb{F}_{q}$, with $p>2$, such that $\gamma_{s}(\mathcal{X})$ is a subcover of a quotient curve $\mathcal{X} / G$, for some linear nontrivial subgroup $G<\operatorname{Aut}(\mathcal{X})$. Then, up to a projective transformation over $\overline{\mathbb{F}}_{q}, \mathcal{X}$ is defined by a polynomial dividing (4.2) and the following hold:

(i) For all $\sigma \in G$, there are $a, b, c \in \overline{\mathbb{F}}_{q}$ (depending on $\sigma$ ) such that

$$
\sigma:(X: Y: Z) \mapsto(a X+b Y+c Z: Y: Z)
$$

(ii) $G=E_{p} \rtimes C$ where $E_{p}$ is an elementary abelian p-group and $C$ is a cyclic group such that $|C|$ divides $\left|E_{p}\right|-1$. Thus in (4.3) we have $a^{\left|E_{p}\right|-1}=1$ and $a=1$ if and only if $\sigma$ has order $p$.

Proof. Since $G$ is nontrivial, from Proposition 4.1, we know that $\mathcal{X}$ is an strange curve. Thus $\mathcal{X}$ is defined by an irreducible factor of (4.2). Since $\overline{\mathbb{F}}_{q}\left(\gamma_{s}(\mathcal{X})\right)=\overline{\mathbb{F}}_{q}\left(z_{2} / z_{1}\right) \subset \overline{\mathbb{F}}_{q}(\mathcal{X})^{G}$, then $\sigma \in G$ fixes $z_{2} / z_{1}$. We conclude that $\sigma(y)=y$ for all $\sigma \in G$. Moreover, since $G$ preserves a generic tangent of $\mathcal{X}$ and all such tangents meet at $(1: 0: 0)$, we obtain that $G$ fixes $(1: 0: 0)$. Therefore, we obtain (4.3)

Finally, considering a generic tangent line $\ell$ to $\mathcal{X}$ (not fixed pointwise by $G$ ), we have that the restriction of $G$ to $\ell$ is an automorphism of $\ell$ fixing the point $P=(1: 0: 0)$. Hence $G$ is isomorphic to a subgroup of the stabilizer of a point on the projective line. Thus it follows from [17, Theorem 1] that $G=E_{p} \rtimes C$ where $E_{p}$ is an elementary abelian $p$-group and $C$ is cyclic with order dividing $\left|E_{p}\right|-1$. The last statement is straightforward.

Example 4.4. If $p=5$, the curve $\mathcal{F}$ given by the affine equation $\left(y x^{3}+2 y\right)^{5} y+\left(x^{3}+2 y\right)^{5}=0$ is irreducible and has order sequence $(0,1,5)$. Such curve satisfies the hypothesis of Theorem 4.3, with the automorphism group generated by $(x, y) \mapsto(\xi x, y)$, where $\xi$ is a primitive cubic root of unity. 
The next result shows that the equation defining the curve $\mathcal{F}$ is example 4.4 is not so specific.

Theorem 4.5. Let $\mathcal{X}$ be plane curve defined over $\mathbb{F}_{q}$, with $p>2$, such that $\gamma_{s}(\mathcal{X})$ is a subcover of a quotient curve $\mathcal{X} / G$, for some linear nontrivial subgroup $G<\operatorname{Aut}(\mathcal{X})$ of order $n$. If $p \nmid|G|$, then up to a projective transformation over $\overline{\mathbb{F}}_{q}, \mathcal{X}$ is defined by an irreducible factor of a polymomial of the type

$$
z_{1}\left(x^{n}, y\right)^{p^{r}} y+z_{2}\left(x^{n}, y\right)^{p^{r}}
$$

with $z_{1}, z_{2} \in \overline{\mathbb{F}}_{q}[x, y], r>0$ and $G=\langle\sigma\rangle$, with $\sigma:(x, y) \mapsto(\xi x, y)$, for a primitive $n$-th root of unity $\xi \in \overline{\mathbb{F}}_{q}$.

Proof. Suppose that $|G|$ is not divisible by $p$. By Theorem 4.3 if $\mathcal{X}: f(x, y)=0$, we have that $\overline{\mathbb{F}}_{q}(\mathcal{X})=$ $\overline{\mathbb{F}}_{q}(x, y)$, there are $z_{1}, z_{2} \in \overline{\mathbb{F}}_{q}(\mathcal{X})$ with $z_{2} / z_{1} \in \overline{\mathbb{F}}_{q}(x, y)^{G}$ such that $z_{1}^{p^{r}} y+z_{2}^{p^{r}}=0$ and $G$ is a cyclic group generated by an automorphism $\sigma:(x, y) \mapsto(a x+b y+c, y)$ with $a \neq 1$. Consider the change of coordinates defined by

$$
T:(x, y) \mapsto(\bar{x}, y)=\left(x+\frac{b}{a-1} y+\frac{c}{a-1}, y\right) .
$$

Then $\tilde{\sigma}:=T \circ \sigma \circ T^{-1}:(\bar{x}, y)=(a \bar{x}, y)$. Moreover, $z_{i}(x, y)=z_{i}\left(\bar{x}-\frac{b}{a-1} y-\frac{c}{a-1}, y\right)=: \tilde{z}_{i}(\bar{x}, y) \in$ $\overline{\mathbb{F}}_{q}(\bar{x}, y)$, and one can check directly that $\tilde{\sigma}\left(\tilde{z_{2}}(\bar{x}, y) / \tilde{z_{1}}(\bar{x}, y)\right)=\tilde{z_{2}}(\bar{x}, y) / \tilde{z_{1}}(\bar{x}, y)$. Now, we clearly have $\overline{\mathbb{F}}_{q}\left(\bar{x}^{n}, y\right) \subset \overline{\mathbb{F}}_{q}(\bar{x}, y)^{G}$, and since $|G|=n$ equality holds. Since $\tilde{z_{2}}(\bar{x}, y) / \tilde{z_{1}}(\bar{x}, y) \in \overline{\mathbb{F}}_{q}(\bar{x}, y)^{G}$, we conclude that $\tilde{z_{2}}(\bar{x}, y) / \tilde{z_{1}}(\bar{x}, y)=z^{\prime}\left(\bar{x}^{n}, y\right)$, for some rational function $z^{\prime}$. Finaly,

$$
y+z^{\prime}\left(\bar{x}^{n}, y\right)^{p^{r}}=y+\frac{z_{2}(x, y)^{p^{r}}}{z_{1}(x, y)^{p^{r}}}=0
$$

Therefore, the result follows from a formal replacement of $\bar{x}$ with $x$ and $z^{\prime}$ with $z_{2} / z_{1}$.

We now give a characterization of equation (4.2) when $p$ divides $|G|$. Let $\mathcal{X}$ and $G$ as in Theorem 4.3, and let $E_{p}<G$ be the $p$-Sylow subgroup of $G$ of order $p^{s}$. Let $m$ be smallest integer for which $G$ is defined over $\mathbb{F}_{p^{m}} \supseteq \mathbb{F}_{q}$. In particular, $s \leq m$, see [17, Theorem 3]. By Theorem 4.3. every element of $E_{p}$ is defined by $\tau_{b, c}:(x, y) \mapsto(x+b y+c, y)$, with $b, c \in \mathbb{F}_{p^{m}}$. Hence $E_{p}$ is isomorphic to the additive group

$$
\Lambda:=\left\{(b, c) \mid \tau_{b, c} \in G\right\}<\mathbb{F}_{p^{m}} \times \mathbb{F}_{p^{m}}
$$

Theorem 4.6. Let $\mathcal{X}$ be plane curve defined over $\mathbb{F}_{q}$, with $p>2$, such that $\gamma_{s}(\mathcal{X})$ is a subcover of a quotient curve $\mathcal{X} / G$, for some linear nontrivial subgroup $G<\operatorname{Aut}(\mathcal{X})$. Suppose that $p$ divides $|G|$ and $\mathbb{F}_{p^{m}}$ is the smallest extension of $\mathbb{F}_{q}$ for which $G$ is defined. Then there exists $n>0$ such that, up to a projective transformation over $\overline{\mathbb{F}}_{q}, \mathcal{X}$ is defined by an irreducible factor of a polynomial of the type

$$
z_{1}\left(\prod_{(b, c) \in \Lambda}(x+b y+c)^{n}, y\right)^{p^{r}} y+z_{2}\left(\prod_{(b, c) \in \Lambda}(x+b y+c)^{n}, y\right)^{p^{r}}
$$


with $z_{1}, z_{2} \in \overline{\mathbb{F}}_{q}[x, y], r>0$ and $\Lambda<\mathbb{F}_{p^{m}} \times \mathbb{F}_{p^{m}}$ is an additive subgroup of $\mathbb{F}_{p^{m}} \times \mathbb{F}_{p^{m}}$ of order $p^{s} \leq p^{m}$ such that $n$ divides $\operatorname{gcd}\left(p^{s}-1, p^{m}-1\right)$. Moreover, $G=E_{p} \rtimes\langle\sigma\rangle$, with $\sigma:(x, y) \mapsto(\xi x, y)$ for a primitive $n$-th root of unity $\xi \in \overline{\mathbb{F}}_{q}$ and $E_{p}=\left\{\tau_{b, c}:(x, y) \mapsto(x+b y+c, y) \mid(b, c) \in \Lambda\right\} \cong \Lambda$ is elementary abelian p-group.

Proof. By Theorem 4.3 and the proof of Theorem 4.5 we may assume that (4.2) holds and $G=E_{p} \rtimes\langle\sigma\rangle$, with $\sigma:(x, y) \mapsto(\xi x, y)$ for a primitive $n$-th root of unity $\xi \in \overline{\mathbb{F}}_{q}, E_{p}$ is elementary abelian $p$-group of order $p^{s}$ and $n \mid p^{s}-1$. In fact, using notation of Theorem 4.5, one can check that $T \circ \tau_{d, e} \circ T^{-1}=\tau_{d, e}$ and $\tau_{d, e}\left(\tilde{z_{i}}(\bar{x}, y)\right)=\tilde{z_{i}}(\bar{x}, y)$ for $i=1,2$. Here, if $m$ is the highest power of $p$ for which $G$ is defined over $\mathbb{F}_{p^{m}}$, we have $q \leq p^{m}$, and by [17, Theorem 3], we have $s \leq m$ and $n \mid p^{m}-1$. In particular, multiplication by $\xi$ gives a faithful action on $\mathbb{F}_{p^{m}}$. Now, the function

$$
w:=\prod_{(b, c) \in \Lambda}(x+b y+c)^{n}
$$

is fixed by every element of $G$. Indeed,

$$
\sigma(w)=\prod_{(b, c) \in \Lambda}(\xi x+b y+c)^{n}=\prod_{(b / \xi, c / \xi) \in \Lambda} \xi^{n}(x+(b / \xi) y+c / \xi)^{n}=w
$$

and

$$
\tau_{d, e}(w)=\prod_{(b, c) \in \Lambda}(x+(b+d) y+(c+e))^{n}=\prod_{(b+d, c+e) \in \Lambda}(x+(b+d) y+(c+e))^{n}=w .
$$

Thus $\overline{\mathbb{F}}_{q}(w, y) \subseteq \overline{\mathbb{F}}_{q}(\mathcal{X})^{G}$. On the other hand, $\left[\overline{\mathbb{F}}_{q}(\mathcal{X}): \overline{\mathbb{F}}_{q}(w, y)\right] \leq p^{s} n=|G|$, and so $\overline{\mathbb{F}}_{q}(w, y)=\overline{\mathbb{F}}_{q}(\mathcal{X})^{G}$. Since $z_{2} / z_{1} \in \overline{\mathbb{F}}_{q}(\mathcal{X})^{G}$, we obtain the claimed result.

Remark 4.7. Let $\mathcal{X}$ be a plane curve of degree d defined over $\overline{\mathbb{F}}_{q}$. We say that $\mathcal{X}$ has controlled singularities if

$$
\sum_{P \in \mathcal{X}} e_{P}<\frac{1}{2} d
$$

where $e_{P}$ denotes the multiplicity of the Jacobian ideal of $\mathcal{X}$ at $P$. In [8, Corollaries 5.10 and 5.16], it is shown that if $\mathcal{X}$ is nonlcassical with order sequence $\left(0,1, p^{r}\right)$ and it has controlled singularities, then $\mathcal{X}$ has an equation of the form (4.1). However, one can check that strange curves does not have controlled singularities. Thus such criteria cannot be applied to our case.

Remark 4.8. Given a plane curve $\mathcal{X}$, a point $P \in \mathbb{P}^{2}\left(\overline{\mathbb{F}}_{q}\right)$ is called a Galois point of $\mathcal{X}$ if the projection $\pi_{P}: \mathcal{X} \longrightarrow \mathbb{P}^{1}\left(\overline{\mathbb{F}}_{q}\right)$ from $P$ induces a Galois extension of function fields $\overline{\mathbb{F}}_{q}(\mathcal{X}) / \overline{\mathbb{F}}_{q}\left(\mathbb{P}^{1}\right)$, see for instance [4. 18]. One can check that if $\overline{\mathbb{F}}_{q}\left(\gamma_{s}(\mathcal{X})\right)=\overline{\mathbb{F}}_{q}(\mathcal{X})^{G}$ for a linear automorphism group $G$, then the nucleus of $\mathcal{X}$ (which exists, since in such case $\mathcal{X}$ is strange) is a Galois point of $\mathcal{X}$. 


\section{Acknowledgements}

This research was partially supported by FAPESP-Brazil, grant 2016/24713-4.

\section{References}

[1] E. Bertini, Introduzione alla geometria proiettiva degli iperspazi, Principato, Messina (1923).

[2] V. Bayer, A. Hefez, Strange curves, Comm. Algebra 19(11) (1991) 3041-3059.

[3] H. Borges, On multi-Frobenius non-classical plane curves, Arch. Math. (Basel) 93(2009), no. 6, 541553.

[4] S. Fukasawa, Complete determination of the number of Galois points for a smooth plane curve, Rend. Semin. Mat. Univ. Padova 129(013) 93-113.

[5] K. Furukawa, A. Ito, On Gauss Maps in positive characteristic in view of images, fibers, and field extensions, Intern. Math. Research Notices, Vol. 2017, No. 8, (2016) 2337-2366.

[6] A. Garcia, J.F. Voloch, Duality for projective curves, Bull. Bras. Math. Society 2 (1991) 159-175.

[7] A. Garcia and J.F. Voloch, Fermat Curves over finite fields, J. Number Theory 30 (1988) 345-356.

[8] A. Hefez, Non-reflexive curves, Compositio Math. 69 (1989) 3-35.

[9] A. Hefez, J.F. Voloch, Frobenius non-classical curves, Arch. Math. 54 (1990), 263-273.

[10] J.W.P. Hirschfeld, G. Korchmáros, Arcs and curves over a finite field, Finite Fields Appl. 5 (1999), 393-408.

[11] J.W.P. Hirschfeld, G. Korchmáros, F. Torres, Algebraic curves over a finite field, Princeton Series in Applied Mathematics, 2008.

[12] M. Homma, H. Kaji, On the inseparable degree of the Gauss map of higher order for space curves, Proc. Japan Acad. 68(1) (1993), 11-14.

[13] H. Kaji, On the space curves with the same image under the Gauss maps, Manuscripta Math. 80 (1993), 249-258.

[14] G. Korchmáros, F. Torres, Embedding of a maximal curve in a Hermitian variety, Composito Math. 128 (2001), 95-113.

[15] H. Stichtenoth, Algebraic function fields and codes, Springer-Verlag, Berlin and Heidelberg, 1993. vii+260 pp. 
[16] K.O. Stöhr, J.F. Voloch, Weierstrass points on curves over finite fields, Proc. London Math. Soc. 52(1986) 1-19.

[17] R. Valentini and M. Madan, A Hauptsatz of L.E. Dickson and Artin-Schreier extensions, Journal für die reine und Angew. Math. 318 (1980), 156-177.

[18] H. Yoshihara, Function field theory of plane curves by dual curves, J. Algebra 239(2001) 340-355. 